\section{Computerization of the operant arena}

\author{
STEPHEN R. GOLDSTEIN \\ Lakehead University, Thunder Bay, Ontario P7B 5E 1, Canada
}

HENRY J. BLEKKENHORST

Great Lakes Forest Products

Thunder Bay, Ontario P7C 4W3, Canada

and

\section{LESLIE W. MAYES \\ Lakehead University. Thunder Bay, Ontario P7B 5E1, Canada}

An operant arena to study schedule control of the distribution and abundance of rats has recently been described (Goldstein, 1981). Eight rats are free to work for food at any one of eight operant food stations. A PET Commodore 2001 series microprocessor controls the experiment while an observer records, on the minute, the number of rats observed at each of the eight stations. (For additional experimental control applications by the PET, see Crossman, Williams, \& Chambers, 1978; Czerny, 1979; McLean, 1978.) This paper describes the computer programs for the schedule functions controlled by the PET and the analysis of the rat dispersion patterns, controlled by the VAX. An interface between the PET and the operant equipment is described by Blekkenhorst and Goldstein (in press).

\section{PET GENERAL PROGRAM DESCRIPTION}

The PET provides simultaneous, independent control over the schedules of reinforcement of all eight stations. It also provides a current update of responses, reinforcements, and momentary status of prevailing schedules on a display that simulates the position of the eight stations in the arena. Further data analyses, including interresponse time (IRT) distribution, mean postreinforcement pause, and the matching relation (Herrnstein, 1970), can also be displayed during or after a session, without interfering with schedule control or data analyses operations. For additional analyses, and as a redundancy measure, all responses and reinforcements are recorded on event recorders that devote two channels to each station and on a cumulative recorder that responds to the input of any combination of all eight stations.

The PET hardware-software work was submitted by H. J. Blekkenhorst to the Faculty of Electrical Engineering in partial fulfillment of the requirements of the Bachelor of Engineering degree. This research was supported in part by the Natural Sciences and Engineering Research Council (NSERC) and the President's NSERC fund to S. R. Goldstein. Reprint requests should be sent to S. R. Goldstein, Department of Psychology, Lakehead University, Thunder Bay, Ontario P7B 5E1, Canada.

\section{SOFTWARE OVERVIEW FOR PET}

Where speed is not critical, such as data display routines, programs are written in BASIC. Where speed is important, such as control and data collection, assembler language is used. The assembler code is completely independent of the BASIC program. This independence is achieved by the vectored interrupt capacity of the PET and works as follows. The BASIC program runs continuously. However, once every $1 / 60 \mathrm{sec}$ (jiffy) the computer branches to, and services, assembler control routines, such as schedule operations. Upon completion of assembler operations, the computer returns to BASIC and begins a new cycle. The only communication between the BASIC and assembler codes is through a common block of memory in which the data are tabulated and control and status information are placed.

\section{Control Algorithm}

The control routines consist of approximately 1,000 words of assembler code. The routines execute at a rate of 60 times/sec after the program is initialized. To direct the control action, control words are written in the common block of memory by the BASIC program. The code corresponding to flowchart decision blocks reads these words and modifies execution flow as required. The data collected are stored in the common block in memory.

The main output function of the control routine is to generate a pulse of a specific duration to a line that fires the dispenser for a particular station. The input function of the control routine scans the input port connected to the bar contacts. The current state of the contacts is compared with the state stored during the last scan, and any changes trigger control routines.

\section{The BASIC Program}

MENU. When the program is idle or when it is first entered, the operator is presented a menu from which a particular function can be chosen. Entering the first letter of any item on the menu causes the program to branch to the desired function.

INITIALIZE. This routine is entered when the user is first starting the program, since it initiates the cyclical execution of the program that controls the arena. The 24-h clock is set, and a reinforcement schedule type is chosen. All eight stations are thereby simultaneously and independently programmed for the schedule entered. The schedule options include interval or ratio, differential reinforcement of short (DRH) or long (DRL) interresponse times, fixed or variable mode, magnitude, and a yoking option.

EXIT. The exit function is the only way to leave the program and to regain control of the keyboard. This 
function prevents interruption of the program by accidental operation of the "STOP" key on the keyboard. The exit function stops the BASIC program, but the control program runs and data collection continues. No data are lost when the program is reentered.

SET SCHEDULES. The set schedule routine allows separate, independent programming of each station. Any schedule option can be changed by deleting and reentering an item.

In the variable mode of operation, the number entered determines a range from which the reset value is chosen. Each time a pellet is delivered at a station, a new reset value is randomly selected from a uniform distribution in the range from $2 n-1$ of the number entered. In the fixed mode, the reset value is always the number entered.

When in the ratio mode of operation, the control program accumulates responses until the number is equal to the reset value. At this time, a pellet is delivered and the data table is updated. In the interval mode, the control program collects responses until the specified interval of time has expired. The resolution time for all time-dependent schedules is $.10 \mathrm{sec}$. When time expires, a reinforcement is delivered on the next response and a new interval is started. The data table is also updated at this time.

The DRL works like the interval schedules except that the timer resets after every response. When the interval between successive responses exceeds the programmed value, a reinforcement is delivered on the terminal response. When the DRH option is selected, reinforcement occurs if an IRT is less than the programmed value.

For the yoking procedure, up to four sets of masterslave pairs are available. The master can be any one of the schedule options. A reinforcement is set for the next response on a slave station at the same time that reinforcement is delivered at the master station.

ARENA. This option will call a display of the data table. It will show all the data collected since the last time a reset was executed. This display is updated every $2 \mathrm{sec}$. The arena is represented by a rectangle, and the eight stations are represented by eight separate data sets corresponding to each station. The data for each station are cumulative responses, cumulative reinforcements, responses this run, and time or response requirement. The arena display also contains the $24 \mathrm{~h}$ clock, which is shown as six digits representing hours, minutes, and seconds.

MATCH. This displays two columns of numbers that represent the relative probability of a response at a station and the corresponding relative probability of reinforcement. The computer determines these values by operating on the following formula: (number of responses at Station $1 /$ number of responses at Stations 1-8) $=$ (number of reinforcements at Station $1 /$ number of reinforcements at Stations 1-8). When session lengths are constant, this formula also provides a measure of relative rate.
GRAPH IRT. This choice results in a histogram display of the IRTs for each station. This display consists of 16 cells, each of which stores the number of times the interval between successive responses is between $2 \exp (\#$ cell) and $2 \exp (\#$ cell -1$) / 60$. The cells are numbered from the right. For example, the center cell, Cell 7, has bounds of 64-128 jiffies and is approximately $1-2 \mathrm{sec}$. The next cell to the left has bounds of approximately $2-4 \mathrm{sec}$. The histogram is normalized so that the largest bar is the full length of the display. The untransformed numerical values are also displayed.

The display showing an IRT histogram also provides a readout of the mean postreinforcement pause for that station. This is derived from a table storing the cumulative pellet-to-postpellet IRT and dividing this value by the number of reinforcements.

RESET. This option allows the data table, operated on by the data collection program, to be cleared. When the arena function is entered from the menu, it will display all zeros. All data collected up to this point will be lost.

A limitation of the present system requires that the relevant data be transcribed to a data book for future use. An advantage is the option of simulating operant responses on any schedule for program testing or behavioral modeling. The PET has been in almost daily operation for about 2 years. During this time, about 1.5 million pellets have been dispensed for some 6 million responses without equipment breakdown or data loss. Complete software listings written for a 16- or 32-KB PET (Version 3.0, 40-character screen) are available on request.

\section{VAX GENERAL PROGRAM DESCRIPTION}

The following software programs deal with the analysis of the spatial dispersion of the rat population and reside on the university's VAX computer.

Dispersion refers to the distribution of animals in space at any one moment (Brown \& Orians, 1970). Dispersal refers to the movements of animals to new locales. In the present context, the distribution of the rats at the bars at any moment is called a dispersion; the change in dispersion from minute to minute defines dispersal.

The raw data represent the number of rats observed at each station, once each minute, for a specified session length, usually $30 \mathrm{~min}$. The data are entered from a standard terminal to an input file in the form of an 8 by 30 matrix. The input files are named by date and time for sequential analysis and long-term storage.

The data analysis software consists of three independent programs and five command language procedures that invoke these routines in combination. The programs consist of over 800 lines of source code written predominantly in standard PASCAL.

The software can be used on a day-by-day basis in "interactive mode," to chart the course of an experiment as new data come in, or in "batch mode," in which 
Table 1

Dispersion, Dispersal, and Density Analyses

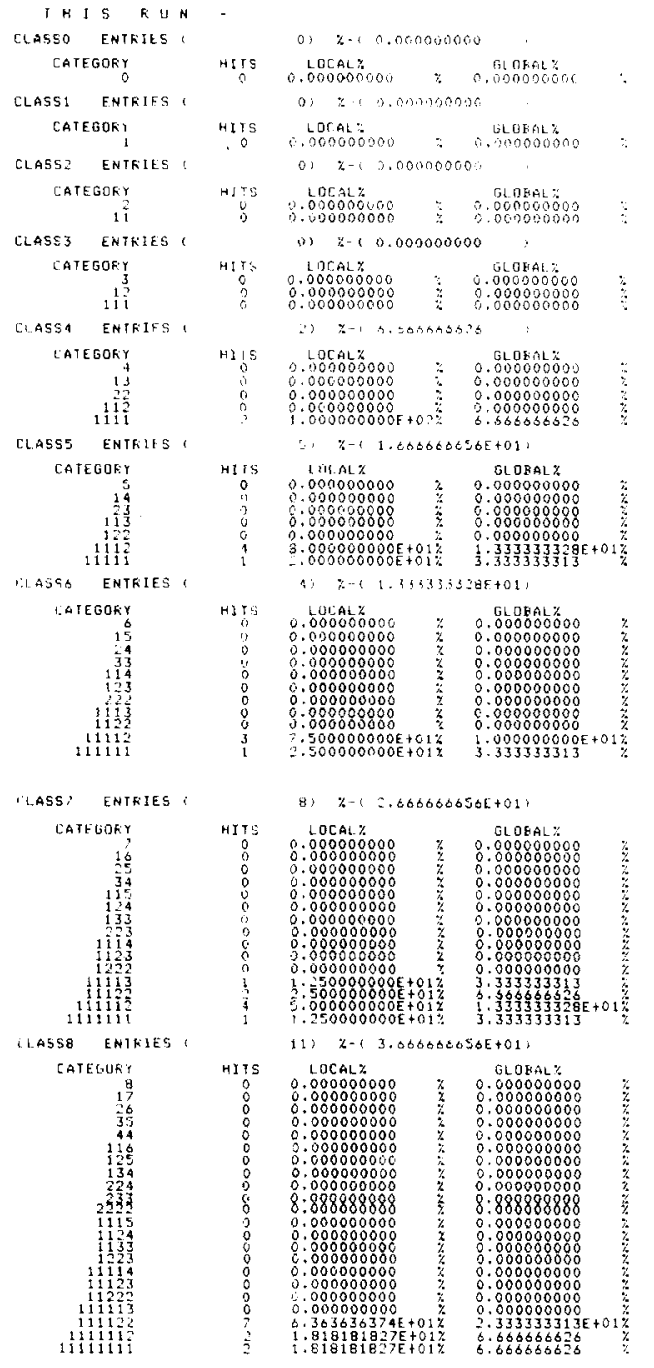

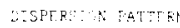
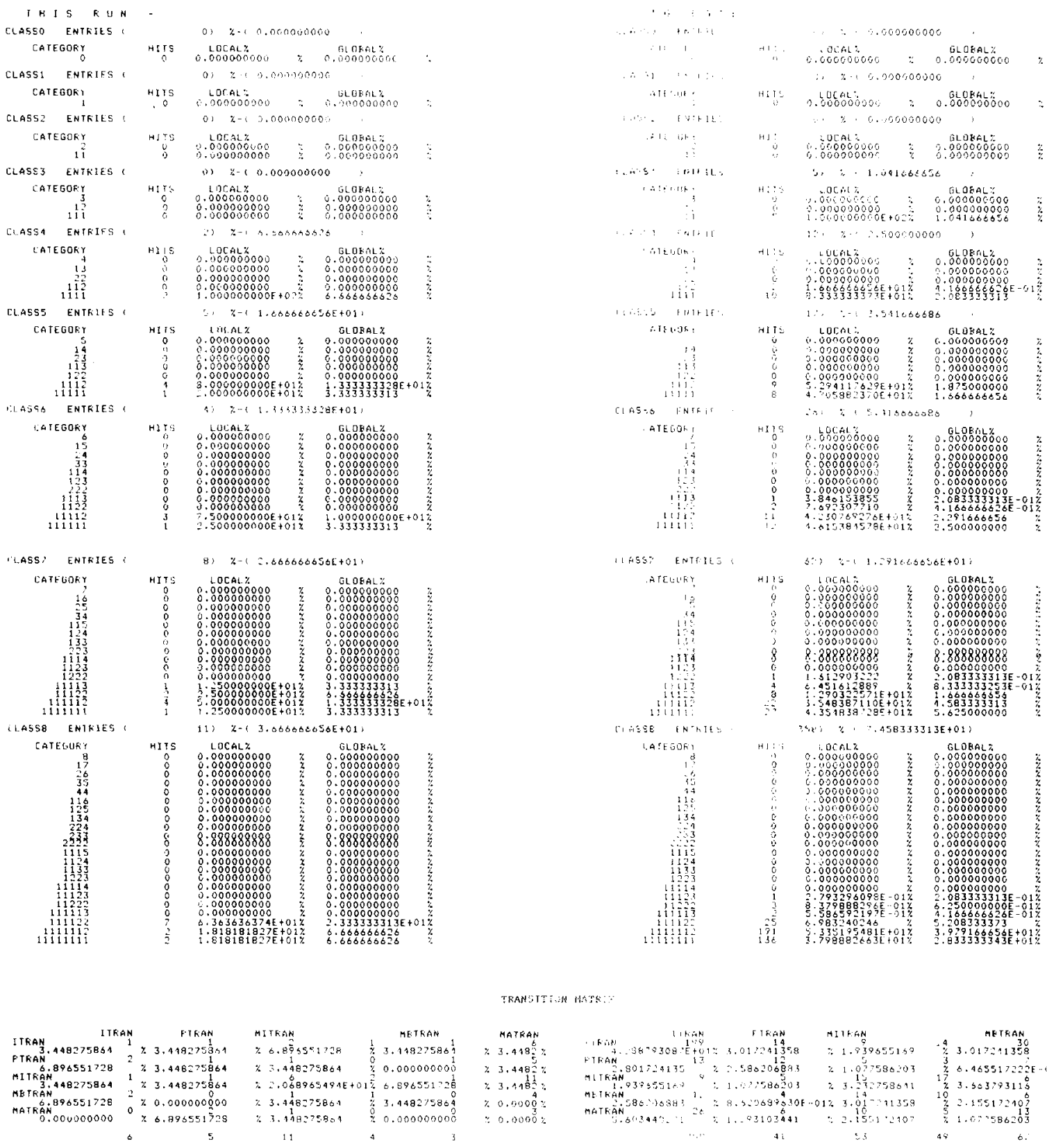

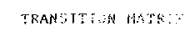

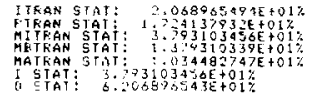

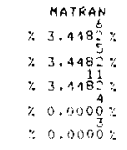
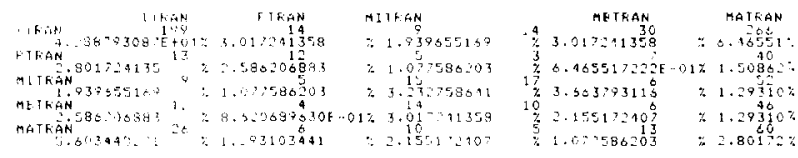

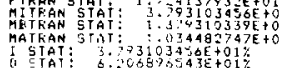

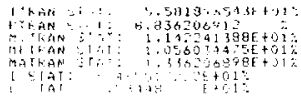

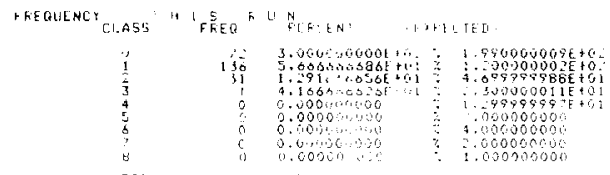

[.M] SOUAKE FOK: 1 H 1 \& 3 A

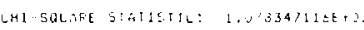

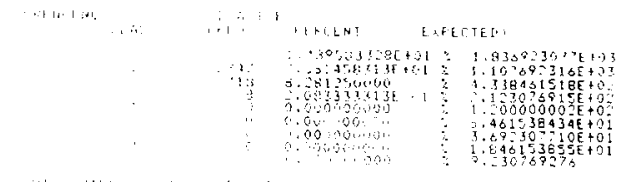

$\therefore .1$ 
any subset of the previously entered data can be chosen for analysis.

The three types of analyses described below are performed on the data for a current session and then again on the cumulative data collected over several sessions until a series or phase of the experiment has been completed. This is done by maintaining a computer file called "To Date," which stores data over a specified number of sessions. The "To Date" file holds the latest cumulative data on dispersion classes, the latest transition matrix, and the latest density data for chi-square analysis.

\section{Dispersion Analysis}

Within the main program, most analyses are performed on a row-by-row basis. Each row is sorted and categorized according to which dispersion pattern is represented (Table 1). Dispersions are further grouped into a "global" and "local" mode to analyze patterns when any combination (global) or when only a specific number (local) of the eight rats work. In this format, a frequency analysis of each type of dispersion can be performed.

\section{Dispersal Analysis}

Since a dispersal is defined as a change in the dispersion pattern from one minute to the next, each pair of rows can be compared and sorted into one of five dispersal types (Goldstein, 1981). An identical transition (ITRAN) denotes a stable condition in which the dispersion patterns remain unchanged from one minute to the next (i.e., 4-0-0-2-1-1-0-0 $\rightarrow$ 4-0-0-2-1-1-0-0). A positional transition (PTRAN) denotes a change in the location of the dispersion pattern (i.e., 4-0-0-2-1-1-0-0 $\rightarrow$ 1-0-0-2-4-1-0-0). A microstate transition (MITRAN) occurs when the same number of rats colonize the same number of stations in different ways (i.e., 4-0-0-2-1-1-0-0 $\rightarrow$ 2-0-0-2-2-2-0-0). A Type A macrostate transition (MATRAN) occurs when the same number of rats colonize a different number of stations in two consecutive minutes (i.e., 4-0-0-2-1-1-0-0 $\rightarrow 5-0-2-0-0-0-1-0$ ), and a Type $B$ macrostate transition (MBTRAN) occurs when different numbers of animals colonize the stations in two consecutive minutes (i.e., 4-0-0-2-1-1-0-0 $\rightarrow$ 4-0-0-1-1-1-0-0).

Acquisition of data in this format permits a frequency analysis of the percentages of each type of dispersal pattern (Table 1). In addition, the format also permits the construction of a transition matrix (Table 1) that represents the likelihood with which each type of change follows another. Minute-by-minute changes in the number of rats observed at a station can also be analyzed by a program operating on the column data. The program provides the sum, mean density, and standard deviation for each of the eight stations.

\section{Density Analysis}

The state of a dispersion pattern can also be analyzed by determining, during any observation frame, whether a station is empty (0), has one rat (1), or up to eight rats (8). The expected frequencies of $0-8$ rats at a station is a Poisson distribution (Poole, 1974); an empirically expected distribution of $0 \mathrm{~s}, 1 \mathrm{~s}, \ldots$, and $8 \mathrm{~s}$ can be directly obtained by counting these in Table 1 . These data can be readily obtained from the present input format structure, and a chi-square analysis of the expected and actual distributions can then be performed.

\section{AVAILABILITY}

A listing of the described programs may be obtained at no cost from Stephen R. Goldstein, Department of Psychology, Lakehead University, Thunder Bay, Ontario P7B 5E1, Canada.

\section{REFERENCES}

Brown, J. L., \& Orians, G. H. Spacing patterns in mobile animals. Annual Review of Ecology and Systematics, 1970, 1, 239-262.

Blekkenhorst, H., \& Goldstein, S. R. A PET interface to control reinforcement delivery. Behavior Research Methods \& Instrumentation, in press.

Crossman, E. K., Williams, J. G., \& Chambers, J. H. Using the PET microcomputer for collecting and analyzing observational data in the classroom. Behavior Research Methods \& Instrumentation, 1978, 10, 563-566.

Czenny, P. Godot: A real-time data acquisition and control program for the PET microcomputer. Behavior Research Methods \& Instrumentation, 1979, 11, 577-580.

Goldstein, S. R. An operant arena for rats. Behavior Research Methods \& Instrumentation, 1981, 13, 37-39.

Herrnstein, R. J. On the law of effect. Journal of the Experimental Analysis of Behavior, 1970, 13, 243-266.

McLeAN, R. S. Using personal computers for experimental control, Behavior Research Methods \& Instrumentation, 1978, 10, 468-473.

Poole, R. W. An introduction to quantitative ecology. New York: McGraw-Hill, 1974.

(Accepted for publication April 12, 1982.) 